\title{
A Parallel Functional Topography between Medial and Lateral Prefrontal Cortex: Evidence and Implications for Cognitive Control
}

\author{
Adrienne A. Taren, ${ }^{1,2 *}$ Vinod Venkatraman, ${ }^{1,2,3 *}$ and Scott A. Huettel ${ }^{1,2,3}$ \\ ${ }^{1}$ Brain Imaging and Analysis Center, ${ }^{2}$ Center for Cognitive Neuroscience, and ${ }^{3}$ Department of Psychology and Neuroscience, Duke University, Durham, \\ North Carolina 27708
}

\begin{abstract}
The dorsomedial and dorsolateral prefrontal cortices (dmPFC and dlPFC) together support cognitive control, with dmPFC responsible for monitoring performance and dIPFC responsible for adjusting behavior. The dIPFC contains a topographic organization that reflects complexity of control demands, with more anterior regions guiding increasingly abstract processing. Recent evidence for a similar gradient within dmPFC suggests the possibility of parallel, hierarchical organization. Here, we measured connectivity between functional nodes of dmPFC and dlPFC using resting-state functional magnetic resonance imaging in humans. We found a posterior-to-anterior connectivity gradient; posterior dmPFC maximally connected to posterior dIPFC and anterior dmPFC maximally connected to anterior dIPFC. This parallel topographic pattern replicated across three independent datasets collected on different scanners, within individual participants, and through both point-to-point and voxelwise analyses. We posit a model of cognitive control characterized by hierarchical interactions - whose level depends on current environmental demands - between functional subdivisions of medial and lateral PFC.
\end{abstract}

\section{Introduction}

A hallmark of the cerebral cortex is its topographic organization. Topographies have been long recognized within sensory and motor systems, whose spatial and somatotopic maps were elucidated in early animal and subsequent human research (Udin and Fawcett, 1988; Reep et al., 1996; Swindale, 1996; Kaas, 1997; Schneider et al., 2004; Silver and Kastner, 2009). Other cortical regions, however, have less obvious large-scale structure. Whereas early conceptions of the prefrontal cortex (PFC) were shaped by the lack of clear functional deficits associated with specific lesions (Ferrier, 1886; Lashley, 1929, 1950; Tizard, 1959), recent theoretical models have argued that all of PFC conjointly supports the adaptive control of behavior (Fuster et al., 2000; Miller and Cohen, 2001).

Within the last decade, functional neuroimaging has generated models of dorsolateral PFC (dlPFC) that contain a topographic organization (Christoff and Gabrieli, 2000; Koechlin et al., 2000, 2003; Christoff and Keramatian, 2007; Koechlin and Summerfield, 2007; Badre and D'Esposito, 2007, Badre, et al., 2008). Although the specific mapping of regions to functions

Received Nov. 2, 2010; revised Jan. 14, 2011; accepted Feb. 2, 2011.

Author contributions: A.A.T., V.V., and S.A.H. designed research; A.A.T. and V.V. analyzed data under the supervision of S.A.H.; A.A.T., V.V., and S.A.H. wrote the paper.

The authors declare no competing financial interests.

${ }^{*}$ A.A.T. and V.V., contributed equally to this work.

This work was supported by National Institute of Neurological Disorders and Stroke Grant P01-41328 and by an Incubator Award from the Duke Institute for Brain Sciences. We thank McKell Carter, John Clithero, and Justin Meyer for assistance with data collection and analysis, and for their manuscript comments. We thank Michael Chee for contribution of data for replication analyses.

Correspondence should be addressed to Scott A. Huettel, Center for Cognitive Neuroscience, Box 90999, Duke University, Durham, NC 27708. E-mail: scott.huettel@duke.edu.

DOI:10.1523/JNEUROSCI.5762-10.2011

Copyright $\odot 2011$ the authors $\quad 0270-6474 / 11 / 315026-06 \$ 15.00 / 0$ differs across models, all share some familial properties: posterior dlPFC controls relatively simple mappings of stimuli to actions, anterior dlPFC shapes complex conditional relationships among behavioral rules, and information flows between regions in a largely hierarchical fashion from anterior to posterior regions.

Considerably less is known about the organization of dorsomedial PFC (dmPFC). It contributes to a welter of cognitive processes, including monitoring performance, selecting actions based on goals, anticipating rewards, and signaling errors and adverse outcomes (Bush et al., 2000; Ridderinkhof et al., 2004a,b). One popular view is that this functional diversity parallels apparent structural heterogeneity, with the above processes considered to be intercalated throughout this region (Ridderinkhof et al., 2004b). Yet, recent evidence from neuroimaging studies suggests that dmPFC has functional, and potentially topographic, subdivisions. Direct comparison of three distinct types of cognitive control demands - response-related, decision-related, and strategy-related-revealed a posterior-to-anterior gradient reflecting the transition from simple to higher-order rules for control (Venkatraman et al., 2009b). Moreover, recent structural analyses indicate that subdivisions of cingulate cortex have distinct whitematter connectivity profiles (Beckmann et al., 2009), particularly with respect to the lateral $\mathrm{PFC}$.

We hypothesized that the dIPFC and dmPFC share a common topographic pattern of functional connectivity, consistent both with the known anatomical connections between these regions (Petrides and Pandya, 1999; Petrides, 2005) and their putative joint contributions to cognitive control (Ridderinkhof et al., 2004a; Egner, 2009; Kouneiher et al., 2009). Here, we mapped functional connectivity within prefrontal cortex using restingstate fMRI (Fransson, 2005; Damoiseaux et al., 2006; De Luca et 
al., 2006; Margulies et al., 2007), drawing data from a large primary dataset and from two independent replications collected on different scanners. Our results demonstrated a clear posterior-toanterior gradient in connectivity: posterior $\mathrm{dmPFC}$ regions were maximally connected to posterior dlPFC regions, whereas anterior $\mathrm{dmPFC}$ regions were maximally connected to anterior dlPFC regions. This parallel topography supports an integrative and hierarchical view of PFC, such that its medial and lateral aspects jointly contribute to the adaptive control of behavior.

\section{Materials and Methods}

Participants and data collection: primary dataset. Sixty-four young adults (32 females; mean age, 24 years; range, $18-43$ years) participated in the primary experiment. All participants received monetary compensation for their participation in the study. Twelve participants were excluded before data analysis due to excessive motion $(>2 \mathrm{~mm})$, leaving a total of 52 participants ( 26 female) in the final analyses. All participants gave written informed consent as part of protocols approved by the Institutional Review Board of Duke University Medical Center.

We report data from a 6 min resting-state scan, which was obtained at the end of a 90 min experimental session. During this scan, participants were instructed to keep their eyes open, to focus on the fixation cross that was presented in the center of the screen, to remain alert, and to refrain from directing their thoughts toward anything specific.

Data were acquired on a 4T GE scanner using an inverse-spiral pulse sequence (Guo and Song, 2003) with the following parameters: repetition time (TR), $2000 \mathrm{~ms}$; echo time (TE), $27 \mathrm{~ms} ; 34$ axial slices parallel to the anterior-posterior commissure (AC-PC) plane, with voxel size of $3.75 \times$ $3.75 \times 3.8 \mathrm{~mm}$. High-resolution 3D full-brain SPGR anatomical images were acquired and used for normalizing individual participants' data.

Participants and data collection: replication datasets. We replicated all analyses in resting-state data from two additional datasets collected on different scanners, with different pulse sequences, and with different groups of participants. Twenty-two young adults (11 female; mean age, 23 years; range, 19-32 years) participated in replication 1. Fifteen young adults ( 5 female; mean age, 22 years; range, $20-24$ years) participated in replication 2. All participants gave written informed consent as part of protocols approved by the Institutional Review Boards of Duke University Medical Center or of the Duke-NUS Graduate Medical School, Singapore.

Replication 1 data were acquired on a 3T GE scanner using a SENSE spiral pulse sequence with the following parameters: TR, $2000 \mathrm{~ms}$; TE, 27 $\mathrm{ms}$; 34 axial slices parallel to the AC-PC plane, with voxel size of $3.75 \times$ $3.75 \times 3.8 \mathrm{~mm}$. High-resolution 3D full-brain SPGR anatomical images were acquired and used for normalizing individual subjects' data. Replication 2 data were acquired on a 3T Siemens scanner using an inverse spiral pulse sequence with the following parameters: TR, $2000 \mathrm{~ms}$; TE, 30 ms; 34 axial slices parallel to the AC-PC plane, with voxel size of $3.75 \times$ $3.75 \times 3.8 \mathrm{~mm}$. High-resolution T1-weighted MPRAGE anatomical images were acquired and used for normalizing individual subjects' data.

Data analyses. We used FEAT (fMRI Expert Analysis Tool) Version 5.92, part of FSL (FMRIB's Software Library; www.fmrib.ox.ac.uk/fsl) package (Smith et al., 2004). Preprocessing consisted of motion correction using MCFLIRT (Motion Correction using fMRIB's Linear Registration Tool), slice timing correction, removal of nonbrain voxels using BET (Brain Extraction Tool), spatial smoothing using a Gaussian kernel with full-width at half maximum $6 \mathrm{~mm}$, and high-pass temporal filtering (cutoff, $100 \mathrm{~s}$ ). Normalization to MNI standard space was performed using FLIRT.

Functional connectivity: selection of seed regions. We identified five seed regions in the $\mathrm{dmPFC}$, hereafter described using the nomenclature DM1 through DM5 (posterior to anterior). The peak coordinates of these seeds were taken from previous work indicating distinct foci for response-, decision-, and strategy-related control (Venkatraman et al., 2009b), which became DM1, DM3, and DM5, respectively. The remaining two seeds, DM2 and DM4, were defined by linear interpolation between the above pairs of neighboring coordinates (Fig. 1, Table 1).

We also identified six seed regions in the dlPFC, hereafter labeled as DL1 through DL6 (posterior to anterior). We identified three of the seed regions' peak coordinates based on prior work delineating a dlPFC to-

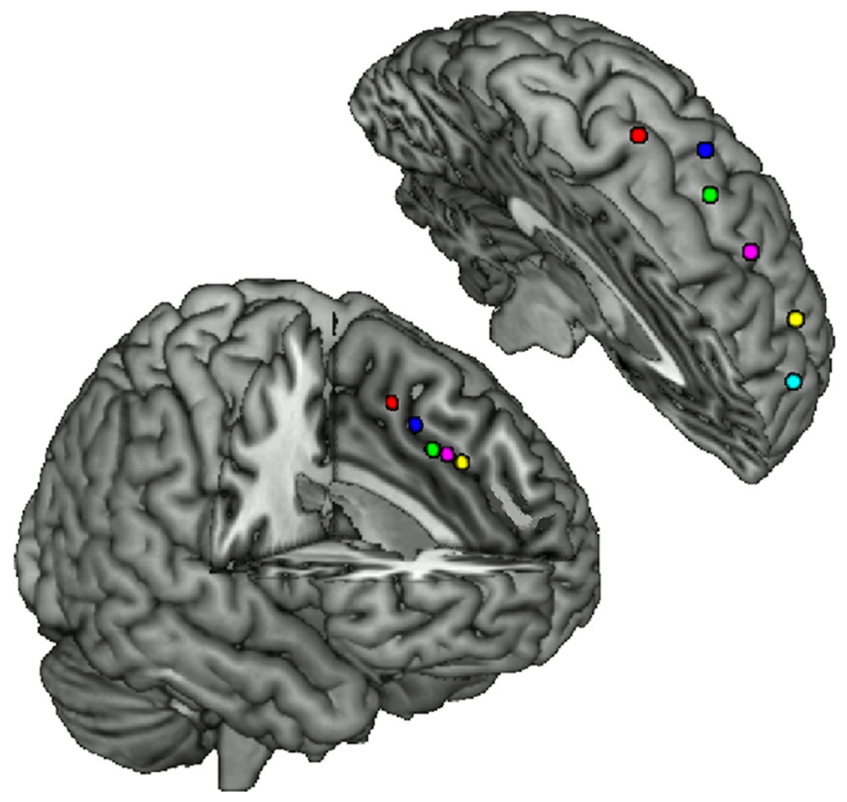

Figure 1. The dmPFC and dIPFC seed regions. The five seed regions within the dmPFC (bottom) and six seed regions within the dIPFC (top) are shown in left hemisphere (see Table 1 for coordinates).

Table 1. MNI coordinates for the dmPFC and dIPFC seed regions

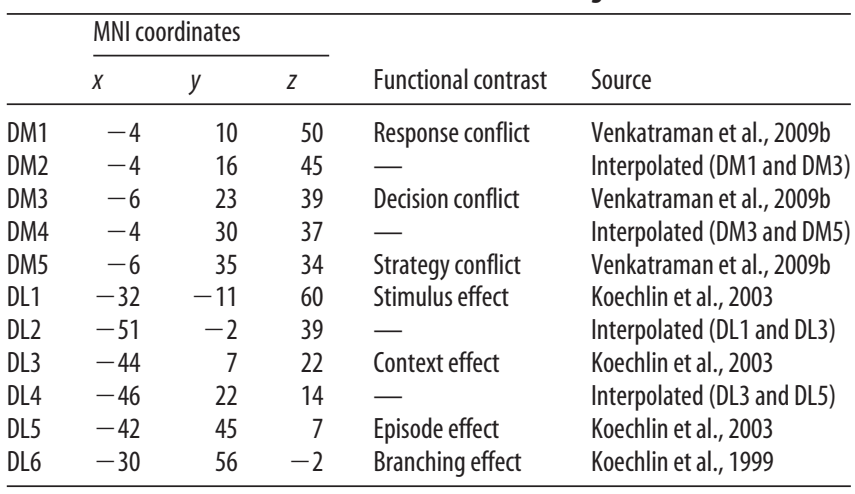

Seed regions were determined based on functional contrasts from previous publications. Coordinates were adapted from the peaks reported in those manuscripts to ensure consistent spacing between adjacent pairings.

pography based on stimulus, context, and integrative demands (Koechlin et al., 2003); these became seeds DL1, DL3, and DL5 (Table 1). The intermediate seeds DL2 and DL4 were defined by linear interpolation between neighboring coordinates and coordinates for DL6 were determined based on prior work associating activation in the anterior prefrontal cortex with cognitive branching (Koechlin et al., 1999). Each seed consisted of $332 \times 2 \times 2 \mathrm{~mm}$ voxels (defined using a sphere with a radius of 2 voxels around the peak coordinate described in Table 1). Time-series signal for all subsequent analyses was obtained by averaging data across all voxels within each seed.

Functional connectivity: data analysis. To map the pattern of functional connectivity between medial and lateral PFC, we began with a dmPFC mask that included the anterior cingulate gyrus and the medial superior frontal gyrus. Following normalization of each participant's data to the standard MNI space, we extracted the time-series from each dmPFC voxel and measured the functional connectivity with each of the six dlPFC seed regions. This provided a voxel-by-voxel map, for each participant, of the correlation between each point in $\mathrm{dmPFC}$ with each of the seed regions in the dlPFC. The corresponding analysis was also performed to obtain a voxel-by-voxel map of the correlation between each point in dIPFC with each of the seed regions in the dmPFC. We used a dlPFC mask, extracted the time-series from each dlPFC voxel, and measured the functional connectivity with each of the five $\mathrm{dmPFC}$ seed regions. 
The above analysis provides a visual representation of topography in dlPFC based on the seeds in dmPFC, and vice versa. To next evaluate the parallel connectivity between hierarchical regions in the $\mathrm{dmPFC}$ and dlPFC, we extracted the preprocessed mean time-series from each of the five $\mathrm{dmPFC}$ seed regions and six dlPFC seed regions for every participant. We demeaned all dmPFC and dlPFC time series by subtracting the mean whole-brain time series (extracted from a whole-brain mask for every participant). To obtain a measure of point-to-point connectivity, we computed Pearson correlations between the five dmPFC time series and each of the six dlPFC time series, which were then combined across all participants (significance tests: Pearson product-moment correlation coefficient, $p<0.05$, two-tailed). All analyses were repeated independently for the left and right hemispheres.

Functional connectivity: specificity analysis. For each dmPFC seed, we calculated the standard deviation (SD) of the correlation values to each of the six dlPFC seeds. A low SD would indicate broad, similar connectivity of voxels within that seed to different regions in the dlPFC, whereas a high SD would indicate greater specificity such that connectivity strength varies across seeds. To statistically test the presence of an anteriorposterior gradient in specificity, we ran an ANOVA of the SD values for each of the five different dmPFC seeds across subjects, followed by post hoc tests of specific seed pairs.

Regression analyses. Each dlPFC/dmPFC correlation for each participant was entered as an observation (i.e., 30 pairwise correlations per participant) into an ordinary least squares regression analysis (JMP Version 7; SAS Institute). Each $\mathrm{dlPFC/dmPFC} \mathrm{correlation} \mathrm{was} \mathrm{served} \mathrm{as} \mathrm{a} \mathrm{depen-}$ dent variable, and explanatory variables included $\mathrm{dmPFC}$ region (normalized posterior to anterior as $-1-1)$, dlPFC region $(-1-1)$, a dmPFC $\mathrm{dlPFC}^{-}$ interaction term, and a categorical variable coding for the dataset from which each observation originated (primary, replication 1 , or replication

2). Using spatial locations as predictors allowed us to answer the question of whether interaction between spatial locations predicts dmPFC-dIPFC correlation (i.e., our measure of connectivity). This analysis was run using the aggregate observations from all three datasets, and was replicated using only the primary dataset.

\section{Results}

We measured the unique, nondirectional functional connectivity between locations within dmPFC and dlPFC using two independent methods, each controlling for the common fMRI signal covariation between the regions as a whole. First, we examined the connectivity between each of the six dlPFC seed regions and every voxel in dmPFC, defined anatomically. The results demonstrate a posterior-to-anterior gradient in connectivity, with posterior dlPFC having maximal connectivity to posterior $\mathrm{dmPFC}$ and anterior dlPFC having maximal connectivity to anterior dmPFC (Fig. 2). The corresponding analysis of connectivity between each of the five dmPFC seed regions and every voxel in dlPFC produces a similar gradient. Second, we examined point-to-point connectivity between the preselected seed regions in dlPFC and dmPFC. We found a similar topographic pattern when focusing on just the con-

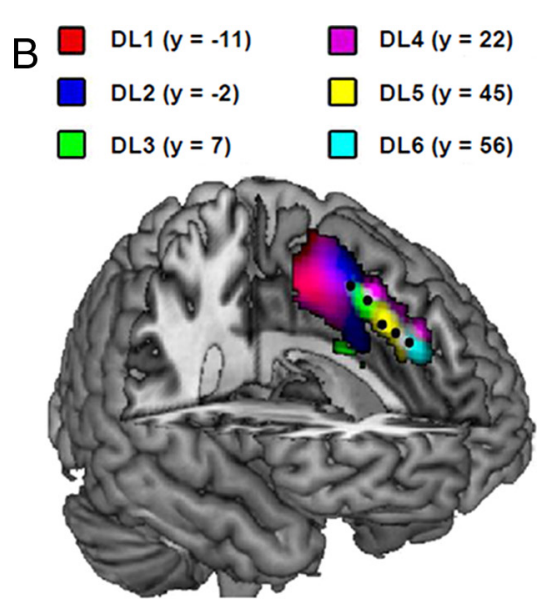

Figure 2. The dmPFC exhibits a topographically organized connectivity pattern with functional regions in dIPFC. A, Voxel-byvoxel correlation between the preselected dmPFC regions of interest and each of the six dIPFC seed regions. For each dIPFC seed gion, the correlated dmPFC voxels are shown in the connecting inset. These maps are thresholded at $p<0.1$ to visualize the distribution of activation. $\boldsymbol{B}$, Overlaying the separate connectivity maps reveals an anterior-to-posterior connectivity gradient
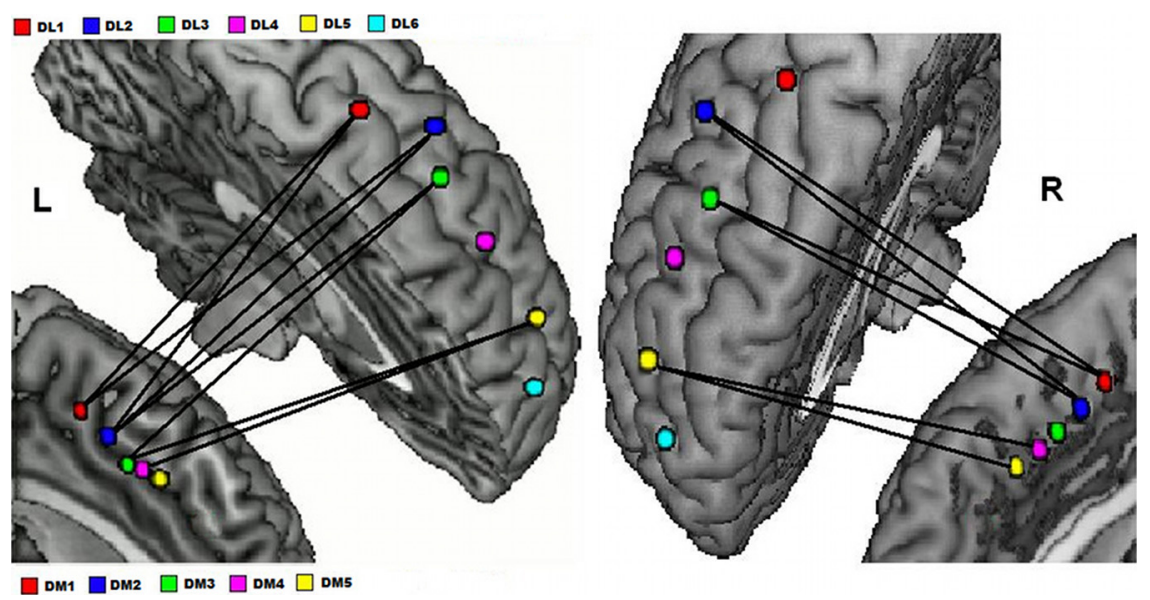

Figure 3. Pairwise connectivity between $\mathrm{dmPFC}$ and dIPFC functional regions. Significant correlations between $\mathrm{dmPFC}$ and dIPFC seed regions in the left and right hemispheres provide evidence for a parallel topography. The lines between seeds indicate pairwise connections that are significant at $p<0.05$.

nectivity between seed regions, independently for both the left and right hemispheres (Fig. 3, Table 2). This topography was replicated in two additional datasets collected on different scanners, with different participants, and using different imaging parameters.

We next evaluated the spatial pattern of functional connectivity using a regression analysis. Point-to-point connectivity values, for all pairs of dmPFC and dlPFC regions and for all subjects, were included as dependent variables in the model. Predictor variables were dmPFC spatial location (five levels), dlPFC spatial location (six levels), and their interaction. Note that this analysis controls for any overall effects of increased connectivity in either posterior or anterior regions. Instead, of primary interest would be a significant interaction, such that connectivity is maximal between regions matched spatially. We found that the $\mathrm{dmPFC}^{\star}$ dlPFC interaction term was highly significant in both the left and right hemispheres (all ps $<10^{-10}$ ) (Table 3). This effect held both for the primary dataset (when considered alone) and for the combination of all three datasets, when including dataset as an additional categorical predictor variable (all $p s<$ $10^{-10}$ in similar analyses to those of Table 3 ). 
Table 2. Pairwise correlations between each $\mathrm{dmPFC}$ and dIPFC seed regions for the left and right hemispheres

\begin{tabular}{|c|c|c|c|c|c|c|c|c|c|c|}
\hline \multicolumn{11}{|c|}{ Primary Dataset } \\
\hline & \multicolumn{5}{|c|}{ Left Hemisphere } & \multicolumn{5}{|c|}{ Right Hemisphere } \\
\hline & DM1 & DM2 & DM3 & DM4 & DM5 & DM1 & DM2 & DM3 & DM4 & DM5 \\
\hline DL1 & 0.45 & 0.24 & 0.19 & 0.10 & 0.08 & 0.26 & 0.19 & 0.11 & 0.04 & 0.00 \\
\hline DL2 & 0.34 & 0.31 & 0.26 & 0.15 & 0.13 & 0.47 & 0.39 & 0.20 & 0.18 & 0.12 \\
\hline DL3 & 0.24 & 0.37 & 0.32 & 0.25 & 0.21 & 0.30 & 0.29 & 0.15 & 0.18 & 0.15 \\
\hline DL4 & 0.11 & 0.25 & 0.21 & 0.24 & 0.25 & 0.17 & 0.12 & 0.14 & 0.17 & 0.16 \\
\hline DL5 & 0.04 & 0.26 & 0.30 & 0.28 & 0.24 & 0.17 & 0.21 & 0.20 & 0.28 & 0.28 \\
\hline DL6 & 0.02 & 0.05 & 0.07 & 0.16 & 0.17 & -0.04 & -0.01 & 0.12 & 0.17 & 0.22 \\
\hline
\end{tabular}

Values labeled in boldface font are individually significant at $p<0.05$.

Table 3. Summary of regression analysis results using data pooled from the primary data set, replication 1 , and replication $2(n=2670)$

\begin{tabular}{lccccc}
\hline & \multicolumn{2}{l}{ Left hemisphere } & & \multicolumn{2}{l}{ Right hemisphere } \\
\cline { 2 - 3 } \cline { 5 - 6 } & $\beta$ & $p$ & & $\beta$ & $p$ \\
\hline Intercept & 0.21 & $<10^{-20}$ & & 0.18 & $<10^{-20}$ \\
dmPFC & -0.02 & 0.01 & & -0.04 & $<10^{-7}$ \\
dIPFC & -0.02 & 0.01 & & 0.01 & 0.04 \\
dmPFC*dIPFC & 0.18 & $<10^{-20}$ & & 0.19 & $<10^{-20}$ \\
\hline
\end{tabular}

Finally, we evaluated whether these findings held for individual participants. We determined, for each participant and each $\mathrm{dmPFC}$ seed, the seed in dIPFC to which there was maximal functional connectivity. This provides a conservative measure of point-to-point connectivity, in that it collapses the entire distribution of connectivity values to a single maximum. As hypothesized, these maxima clustered along the diagonal, consistent with a parallel topography at the individual level in both hemispheres (Fig. 4). A similar pattern was seen in our two replications.

We additionally observed that the specificity of the functional connectivity decreased when moving along the posterior-toanterior gradient (Fig. 4) within the dmPFC. We computed the SD of connectivity values from each dmPFC seed to the set of the six dlPFC seeds. There was a significant decrease from posterior to anterior dmPFC seeds $\left(F_{(4,255)}=2.77, p=0.02\right)$. Voxels in posterior $\mathrm{dmPFC}$ exhibited greater specificity $($ mean $=0.23)$ in connectivity to dlPFC seeds than did voxels in anterior dmPFC $\left(\right.$ mean $\left.=0.18, t_{(51)}=3.20, p=0.002\right)$.

\section{Discussion}

Several lines of empirical evidence have suggested a hierarchical rostral-to-caudal organization within the dlPFC, with neurons in the more rostral regions processing more abstract goals and neurons in the more posterior regions processing more concrete information related to corresponding motor actions (Fuster et al., 2000; Fuster, 2001, 2004; Koechlin and Summerfield, 2007). More recent evidence also suggests a similar anterior-to-posterior topography within the $\mathrm{dmPFC}$, with the more anterior regions being associated with more complex and abstract levels of cognitive control (Venkatraman et al., 2009a,b). Given the strong anatomical evidence for reciprocal connections between these regions based on studies in nonhuman primates (Bates and Goldman-Rakic, 1993; Petrides and Pandya, 1999), a natural hypothesis is that monitoring-related activity in $\mathrm{dmPFC}$ engages regulatory processes in the dlPFC that shape behavior (Botvinick et al., 2001, 2008; Kerns et al., 2004). Yet, despite the striking similarity in their hierarchical organization, it has been unclear whether a similar gradient also exists in the functional connectivity between these regions.

In one recent study, Kouneiher and colleagues (2009) demonstrated that the medial prefrontal cortex regulates processing re- sources in the lateral PFC according to motivational incentives. Specifically, there is increased effective connectivity between posterior dmPFC [pre-supplementary motor area (pre-SMA)] and posterior dlPFC for contextual incentives and increased connectivity between middle dmPFC and mid-dlPFC for episodic incentives. However, there was no increased connectivity during control conditions that lacked such incentives. Therefore, the authors argue for a posterior-to-anterior hierarchical system of executive processes in the medial and lateral PFC with the functional interactions between them conveying motivational incentives rather than simple control demands.

In the current study, we used spontaneous fluctuations in blood oxygenation level-dependent (BOLD) activity to characterize further the functional connectivity between the medial and lateral prefrontal regions in the absence of task-associated cognitive processes. This task-free approach using resting-state data relies on correlations among low-frequency BOLD changes to identify regions that function in tandem. Using such an approach, we demonstrated a posterior-to-anterior gradient in connectivity between the medial and lateral prefrontal regions, with posterior dlPFC having maximal connectivity to posterior $\mathrm{dmPFC}$ and anterior dlPFC to anterior $\mathrm{dmPFC}$. This pattern replicated in three independent datasets collected using three different MR scanners and was evident even in individual participants.

This pattern of resting-state functional connectivity is consistent with prior mappings of structural connectivity between these regions in both nonhuman primates (Petrides and Pandya, 1999) and humans (Beckmann et al., 2009). Petrides and Pandya (1999) used florescent tracers placed in selected areas of the dorsolateral frontal cortex of monkeys to study their connectivity to the rest of the brain. They found that injections placed in posterior dlPFC (similar to DL1 in our study) revealed labeled neurons in posterior dmPFC regions of the SMA, pre-SMA, and cingulate sulcus (similar to DM1 in our study). In contrast, injections placed in middlPFC (corresponding approximately to DL3 seed in our study) revealed labeled cells in a more anterior part of cingulate cortex (here, approximately DM3). Similar connection patterns in the medial regions were also observed for injections placed in rostrolateral dlPFC (corresponding to DL6 seed in our study). These findings suggest strong intrinsic structural connectivity between lateral and medial prefrontal regions in the macaque cortex.

In another study involving magnetic resonance tractographybased parcellation of the human cingulate cortex (Beckmann et al., 2009), the dorsolateral prefrontal regions (consistent with seeds DL3 and DL4 in our study) showed greatest connectivity with anterior cingulate sulcus (similar to DM4 in our study), whereas the premotor regions (overlapping with our seeds DL1 and DL2) showed greatest connectivity with the pre-SMA and more posterior regions within the cingulate (overlapping with seeds DM1 and DM2). Therefore, these studies provide indepen- 
Left Hemisphere

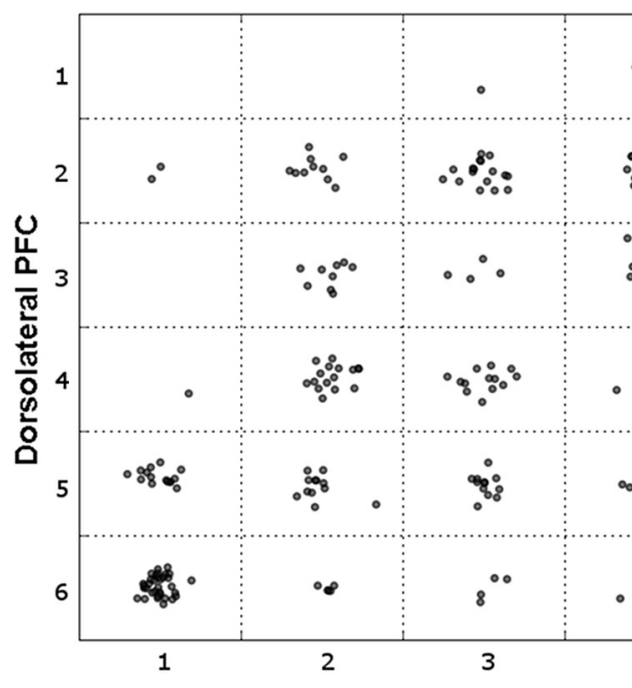

Right Hemisphere

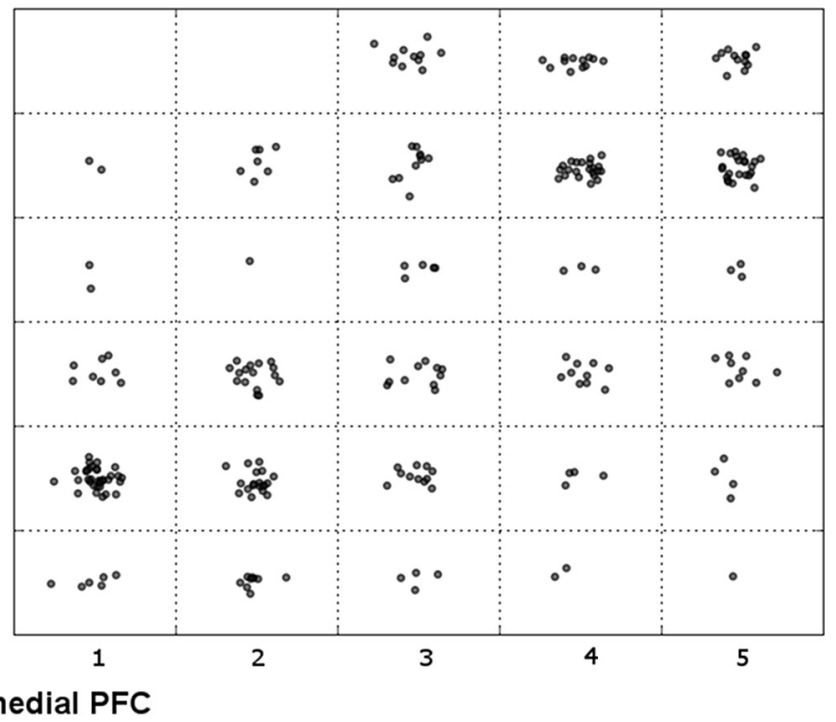

Figure 4. Individual participants' maximally correlated dmPFC-dIPFC pairs reveal parallel topography. Each cell contains one dot for each participant whose maximal connectivity with a given dmPFC seed ( $x$-axis) was to a given dIPFC seed ( $y$-axis). Dots are spatially jittered within a cell for clarity. Even with this very conservative approach-which only considers the single maximal connection, without regard for the distribution - there is an increase in density along the diagonal.

dent evidence across different modalities about the robustness of the functional connections between these regions. Though these methods are largely agnostic about the directionality of these interactions, the findings of Kouneiher and colleagues (2009) suggest that the medial frontal regions may regulate the cognitive control resources in the lateral regions according to motivational incentives. Therefore, we speculate that dmPFC exerts a regulatory or modulatory influence on dlPFC (Wood and Grafman, 2003; Egner, 2009; Kouneiher et al., 2009).

We contend that different regions of dmPFC and dlPFC, according to the computational demands of the task, interact to guide the adaptive control of behavior (Fig. 5). The proposed model extends the hierarchical organization postulated for dlPFC to include a parallel organization in dmPFC, whose subregions shape processing in their lateral counterparts. That is, the anterior dmPFC regulates activity in the anterior dIPFC when the control demands are associated with high levels of abstraction (e.g., implementing strategic planning in a decision-making task), whereas the posterior dmPFC works in concert with posterior dlPFC and premotor cortices when the control demands are limited to choosing between two competing responses. Although we emphasize potential medial-to-lateral effects, bidirectional influences are likely; dmPFC activity may be biased by dlPFC inputs, consistent with the former's role in monitoring the reward value of action sets according to outcomes (Daw et al., 2006; Kennerley et al., 2006). Moreover, whether these regions interact in a hierarchical fashion remains a provocative question. Anterior regions of dlPFC influence processing in posterior regions of dlPFC in a task-dependent manner (Koechlin et al., 2003; Kouneiher et al., 2009), and damage to more anterior portions of prefrontal cortex leads to deficits on a wider array of tasks, including abstract processing (Badre et al., 2009). Our finding that the more anterior regions in the $\mathrm{dmPFC}$ demonstrate more diffuse projections to the lateral PFC, relative to the posterior regions, provides converging evidence toward a hierarchical perspective.

The new framework proposed here provides empirical evidence for a longstanding conjecture: that the PFC contributes to the temporal integration of behavior by simultaneously main- 
taining context and goal-related information at several levels (Fuster, 2001, 2004). Fuster $(2001 ; 2004)$ argued that automatic behaviors only require integration at lower levels—sensory areas in the posterior perceptual hierarchy and motor areas in the frontal executive hierarchy-whereas more complex behaviors require integration at higher levels of perceptual and executive hierarchies. This influential conjecture has recently gained significant supporting evidence in the lateral PFC (Koechlin and Summerfield, 2007; Badre and D'Esposito, 2009). We show that this topography extends to the functional interactions between the medial and lateral PFC. Such a functional gradient in connectivity could reflect a dynamic mechanism for identifying and responding adaptively to contextual changes in behavior.

\section{References}

Badre D (2008) Cognitive control, hierarchy, and the rostro-caudal organization of the frontal lobes. Trends Cogn Sci 12:193-200.

Badre D, D'Esposito M (2007) Functional magnetic resonance imaging evidence for a hierarchical organization of the prefrontal cortex. J Cogn Neurosci 19:2082-2099.

Badre D, D'Esposito M (2009) Is the rostro-caudal axis of the frontal lobe hierarchical? Nat Rev Neurosci 10:659-669.

Badre D, Hoffman J, Cooney JW, D’Esposito M (2009) Hierarchical cognitive control deficits following damage to the human frontal lobe. Nat Neurosci 12:515-522.

Bates JF, Goldman-Rakic PS (1993) Prefrontal connections of medial motor areas in the rhesus monkey. J Comp Neurol 336:211-228.

Beckmann M, Johansen-Berg H, Rushworth MF (2009) Connectivity-based parcelation of human cingulate cortex and its relation to functional specialization. J Neurosci 29:1175-1190.

Botvinick MM (2008) Hierarchical models of behavior and prefrontal function. Trends Cogn Sci 12:201-208.

Botvinick MM, Braver TS, Barch DM, Carter CS, Cohen JD (2001) Conflict monitoring and cognitive control. Psychol Rev 108:624-652.

Bush G, Luu P, Posner MI (2000) Cognitive and emotional influences in anterior cingulate cortex. Trends Cogn Sci 4:215-222.

Christoff K, Gabrieli JD (2000) The frontopolar cortex and human cognition: evidence for a rostrocaudal hierarchical organization within the human prefrontal cortex. Psychobiology 28:168-186.

Christoff K, Keramatian K (2007) Abstraction of mental representations: theoretical considerations and neuroscientific evidence. In: Perspectives on rule-guided behavior (Bunge SA, Wallis JD, eds). Oxford: Oxford UP.

Damoiseaux JS, Rombouts SA, Barkhof F, Scheltens P, Stam CJ, Smith SM, Beckmann CF (2006) Consistent resting-state networks across healthy subjects. Proc Natl Acad Sci U S A 103:13848-13853.

Daw ND, O'Doherty JP, Dayan P, Seymour B, Dolan RJ (2006) Cortical substrates for exploratory decisions in humans. Nature 441:876-879.

De Luca M, Beckmann CF, De Stefano N, Matthews PM, Smith SM (2006) fMRI resting state networks define distinct modes of long-distance interactions in the human brain. Neuroimage 29:1359-1367.

Egner T (2009) Prefrontal cortex and cognitive control: motivating functional hierarchies. Nat Neurosci 12:821-822.

Ferrier D (1886) The functions of the brain, 2nd edition. London: Smith Elder.

Fransson P (2005) Spontaneous low-frequency BOLD signal fluctations: an fMRI investigation of the resting-state default mode of brain function hypothesis. Hum Brain Mapp 26:15-29.

Fuster JM (2001) The prefrontal cortex: an update: time is of the essence. Neuron 30:319-333.

Fuster JM (2004) Upper processing stages of the perception-action cycle. Trends Cogn Sci 8:143-145.

Fuster JM, Bodner M, Kroger JK (2000) Cross-model and cross-temporal association in neurons of frontal cortex. Nature 405:347-351.

Guo H, Song AW (2003) Spiral-in-and-out functional image acquisition with embedded $z$-shimming for susceptibility signal recovery. J Magn Reson Imaging 18:389-395.
Kaas JH (1997) Topographic maps are fundamental to sensory processing. Brain Res Bull 44:107-112.

Kennerley SW, Walton ME, Behrens TE, Buckley MJ, Rushworth MF (2006) Optimal decision making and the anterior cingulate cortex. Nat Neurosci 9:940-947.

Kerns JG, Cohen JD, MacDonald AW 3rd, Cho RY, Stenger VA, Carter CS (2004) Anterior cingulate conflict monitoring and adjustments in control. Science 303:1023-1026.

Koechlin E, Summerfield C (2007) An information theoretical approach to prefrontal executive function. Trends Cogn Sci 11:229-235.

Koechlin E, Basso G, Pietrini P, Panzer S, Grafman J (1999) The role of the anterior prefrontal cortex in human cognition. Nature 399:148-151.

Koechlin E, Corrado G, Pietrini P, Grafman J (2000) Dissociating the role of the medial and lateral anterior prefrontal cortex in human planning. Proc Natl Acad Sci U S A 97:7651-7656.

Koechlin E, Ody C, Kouneiher F (2003) The architecture of cognitive control in the human prefrontal cortex. Science 302:1181-1185.

Kouneiher F, Charron S, Koechlin E (2009) Motivation and cognitive control in the human prefrontal cortex. Nat Neurosci 12:939-945.

Lashley KS (1929) Brain mechanisms and intelligence: a quantitative study of injuries to the brain. Chicago: University of Chicago.

Lashley KS (1950) In search of engram. Society Exper Biol Symp 4:454-482.

Margulies DS, Kelly AM, Uddin LQ, Biswal BB, Castellanos FX, Milham MP (2007) Mapping the functional connectivity of anterior cingulate cortex. Neuroimage 37:579-588.

Miller EK, Cohen JD (2001) An integrative theory of prefrontal cortex function. Annu Rev Neurosci 24:167-202.

Petrides M (2005) Lateral prefrontal cortex: architectonic and functional organization. Philos Trans R Soc Lond B Biol Sci 360:781-795.

Petrides M, Pandya DN (1999) Dorsolateral prefrontal cortex: comparative cytoarchitectonic analysis in the human and the macaque brain and corticocortical connection patterns. Eur J Neurosci 11:1011-1036.

Reep RL, Corwin JV, King V (1996) Neuronal connections of orbital cortex in rats: topography of cortical and thalamic afferents. Exp Brain Res 111:215-232.

Ridderinkhof KR, van den Wildenberg WP, Segalowitz SJ, Carter CS (2004a) Neurocognitive mechanisms of cognitive control: the role of prefrontal cortex in action selection, response inhibition, performance monitoring, and reward-based learning. Brain Cogn 56:129-140.

Ridderinkhof KR, Ullsperger M, Crone EA, Nieuwenhuis S (2004b) The role of the medial frontal cortex in cognitive control. Science 306:443-447.

Schneider KA, Richter MC, Kastner S (2004) Retinotopic organization and functional subdivisions of the human lateral geniculate nucleus: a highresolution functional magnetic resonance imaging study. J Neurosci 24:8975-8985.

Silver MA, Kastner S (2009) Topographic maps in human frontal and parietal cortex. Trends Cogn Sci 13:488-495.

Smith SM, Jenkinson M, Woolrich MW, Beckmann CF, Behrens TE, Johansen-Berg H, Bannister PR, De Luca M, Drobnjak I, Flitney DE, Niazy RK, Saunders J, Vickers J, Zhang Y, De Stefano N, Brady JM, Matthews PM (2004) Advances in functional and structural MR image analysis and implementation as FSL. Neuroimage 23 [Suppl 1]:S208-S219.

Swindale NV (1996) The development of topography in the visual cortex: a review of models. Network 7:161-247.

Tizard B (1959) Theories of brain localization from Flourens to Lashley. Med Hist 3:132-145

Udin SB, Fawcett JW (1988) Formation of topographic maps. Annu Rev Neurosci 11:289-327.

Venkatraman V, Payne JW, Bettman JR, Luce MF, Huettel SA（2009a） Separate neural mechanisms underlie choices and strategic preferences in risky decision making. Neuron 62:593-602.

Venkatraman V, Rosati AG, Taren AA, Huettel SA (2009b) Resolving response, decision, and strategic control: evidence for a functional topography in dorsomedial prefrontal cortex. J Neurosci 29:13158-13164.

Wood JN, Grafman J (2003) Human prefrontal cortex: processing and representational perspectives. Nat Rev Neurosci 4:139-147. 